\title{
Inhibition of skin tumor growth and angiogenesis in vivo by activation of cannabinoid receptors
}

\author{
M. Llanos Casanova, ${ }^{1}$ Cristina Blázquez, ${ }^{2}$ Jesús Martínez-Palacio, ${ }^{1}$ \\ Concepción Villanueva, ${ }^{3}$ M. Jesús Fernández-Aceñero, ${ }^{3}$ John W. Huffman, ${ }^{4}$ \\ José L. Jorcano, ${ }^{1}$ and Manuel Guzmán ${ }^{2}$ \\ ${ }^{1}$ Project on Cellular and Molecular Biology and Gene Therapy, Centro de Investigaciones Energéticas, \\ Medioambientales y Tecnológicas, Madrid, Spain \\ ${ }^{2}$ Department of Biochemistry and Molecular Biology I, School of Biology, Complutense University, Madrid, Spain \\ ${ }^{3}$ Department of Pathology, Hospital General de Móstoles, Madrid, Spain \\ ${ }^{4}$ Department of Chemistry, Clemson University, Clemson, South Carolina, USA
}

\begin{abstract}
Nonmelanoma skin cancer is one of the most common malignancies in humans. Different therapeutic strategies for the treatment of these tumors are currently being investigated. Given the growthinhibiting effects of cannabinoids on gliomas and the wide tissue distribution of the two subtypes of cannabinoid receptors $\left(\mathrm{CB}_{1}\right.$ and $\left.\mathrm{CB}_{2}\right)$, we studied the potential utility of these compounds in anti-skin tumor therapy. Here we show that the $\mathrm{CB}_{1}$ and the $\mathrm{CB}_{2}$ receptor are expressed in normal skin and skin tumors of mice and humans. In cell culture experiments pharmacological activation of cannabinoid receptors induced the apoptotic death of tumorigenic epidermal cells, whereas the viability of nontransformed epidermal cells remained unaffected. Local administration of the mixed $\mathrm{CB}_{1} / \mathrm{CB}_{2}$ agonist WIN-55,212-2 or the selective $\mathrm{CB}_{2}$ agonist JWH-133 induced a considerable growth inhibition of malignant tumors generated by inoculation of epidermal tumor cells into nude mice. Cannabinoid-treated tumors showed an increased number of apoptotic cells. This was accompanied by impairment of tumor vascularization, as determined by altered blood vessel morphology and decreased expression of proangiogenic factors (VEGF, placental growth factor, and angiopoietin 2). Abrogation of EGF-R function was also observed in cannabinoid-treated tumors. These results support a new therapeutic approach for the treatment of skin tumors.
\end{abstract}

J. Clin. Invest. 111:43-50 (2003). doi:10.1172/JCI200316116.

\section{Introduction}

The epidermis is a stratified squamous epithelium composed mainly of keratinocytes, whose proliferation and differentiation must be tightly regulated and coordinated. Basal keratinocytes, which are attached to the basement membrane, are undifferentiated and have proliferative potential. Before entering the differentiation program, they withdraw from the cell cycle and migrate toward the surface of the epidermis, leading to the formation of the outermost layer of the epidermis composed of enucleated dead squames, which are continuously shed from the surface of the skin (1). The incidence of both benign and malignant skin neoplasms has been rising at an alarming rate for the past several years. Thus, nonmelanoma skin cancer is one of the most common malignancies in humans: basal cell carcinomas and squa-

Received for publication June 7, 2002, and accepted in revised form November 19, 2002.

Address correspondence to: Manuel Guzmán, Department of Biochemistry and Molecular Biology I, School of Biology,

Complutense University, 28040 Madrid, Spain.

Phone: 34-913944668; Fax: 34-913944672;

E-mail: mgp@bbm1.ucm.es.

Conflict of interest: The authors have declared that no conflict of interest exists.

Nonstandard abbreviations used: placental growth factor (PIGF); angiopoietin 1 (Ang1); squamous cell carcinoma (SCC). mous cell carcinomas represent the vast majority of the malignant tumors diagnosed (2). These tumors are believed to arise mainly from stem cells of hair follicles (3), and their growth and development seems to rely on an early burst of neovascularization (4) in which VEGF $(5-7)$ and EGF-R $(8,9)$ are essential components. The skin is also a major site for metastasis of internal disease $(2,10)$. Early recognition, biopsy confirmation, and treatment selection can reduce patient morbidity. Different types of strategies are currently being investigated as therapies for the treatment of these tumors, including cryotherapy, topical chemotherapeutic agents such as 5-fluorouracil, and photodynamics, the success of which is hampered by limitations such as the poor penetration of molecules into the skin and the difficulty to gain access to the whole tumor (10-12).

Cannabinoids, the active components of Cannabis sativa linnaeus (marijuana) and their derivatives, exert a wide array of effects on the CNS as well as on peripheral sites such as the immune, cardiovascular, digestive, reproductive, and ocular systems (13-15). Nowadays, it is widely accepted that most of these effects are mediated by the activation of specific $G$ protein-coupled receptors that are normally bound by a family of endogenous ligands - the endocannabinoids $(14,16$, 17). Two different cannabinoid receptors have been characterized and cloned from mammalian tissues: the 
"central" $\mathrm{CB}_{1}$ receptor, mostly expressed in brain and responsible for cannabinoid psychoactivity (18), and the "peripheral" $\mathrm{CB}_{2}$ receptor, mostly expressed in the immune system and unrelated to cannabinoid psychoactivity (19). Marijuana and its derivatives have been used in medicine for many centuries, and currently there is a renaissance in the study of the therapeutic effects of cannabinoids, which constitutes a widely debated issue with ample scientific and social relevance. Ongoing research is determining whether cannabinoid ligands may be effective agents in the treatment of, for example, pain and inflammation, neurodegenerative disorders such as multiple sclerosis and Parkinson's disease, and the wasting and emesis associated with AIDS and cancer chemotherapy (13-15). In addition, cannabinoids may be potential antitumoral agents owing to their ability to induce the regression of various types of tumors, including lung adenocarcinoma (20), glioma $(21,22)$, and thyroid epithelioma (23) in animal models. Although cannabinoids directly induce apoptosis or cell cycle arrest in different transformed cells in vitro (24), the involvement of this and other potential mechanisms (e.g., inhibition of tumor angiogenesis) in their antitumoral action in vivo is as yet unknown.

This background prompted us to explore whether (a) the skin and skin tumors express cannabinoid receptors; (b) cannabinoid receptor activation exerts a growth-inhibiting action on skin tumors in vivo; and (c) inhibition of angiogenesis is implicated in the antitumoral effect of cannabinoids. Our data show that (a) $\mathrm{CB}_{1}$ and $\mathrm{CB}_{2}$ receptors are present in the skin and skin tumors; (b) local cannabinoid receptor activation induces the regression of skin tumors in vivo; and (c) at least two mechanisms may be involved in this action: direct apoptosis of tumor cells and inhibition of tumor angiogenesis.

\section{Methods}

Cannabinoids. JWH-133 was prepared in J.W. Huffman's laboratory (25). WIN-55,212-2 was from Sigma-Aldrich (St. Louis, Missouri, USA). SR141716 and SR144528 were kindly given by Sanofi-Synthelabo (Montpellier, France).

Cell culture. The mouse tumorigenic epidermal cell lines PDV.C57 and $\mathrm{HaCa} 4$, and the nontransformed epidermal cell lines MCA3D (mouse) and HaCat (human), were routinely maintained in DMEM supplemented with $10 \%$ FCS. Twenty-four hours before the experiments, cells were transferred to low serum $(0.5 \%)$ DMEM. Primary human keratinocytes (Biowhittaker Europe SPRL, Vervier, Belgium) were grown and cultured for the experiments in KGM-2 medium (Biowhittaker Europe SPRL). Stock solutions of cannabinoid ligands were prepared in DMSO. Control incubations had the corresponding DMSO content. No significant influence of DMSO was observed on cell viability at the final concentration used $(0.1-0.2 \%, \mathrm{vol} / \mathrm{vol})$.

\section{Table 1}

Quantification of apoptotic and proliferative cells in vehicle- and cannabinoid-treated skin carcinomas

\begin{tabular}{lccc}
\hline & Control & JWH & \multicolumn{1}{c}{ WIN } \\
TUNEL (positive cells/field) & $2.6 \pm 1.1$ & $7.2 \pm 3.6^{\mathrm{B}}$ & $5.6 \pm 2.5^{\mathrm{B}}$ \\
BrdU (positive cells/section) & $15.0 \pm 8.7$ & $23.8 \pm 15.2$ & $10.3 \pm 4.7$
\end{tabular}

Tumor-bearing mice were treated with vehicle (Control), WIN-55,212-2 (WIN), or JWH-133 (JWH) for 11 days, and then tumors were dissected. Several sections of five different tumors were analyzed per group. APositive cells per ten high-power fields. ${ }^{B}$ Significantly different $(P<0.05)$ from control mice.

Human tumor samples. Formalin-fixed, paraffinembedded, human tumor samples were obtained from the files of the Department of Pathology, Hospital General de Móstoles (Madrid, Spain). Six-micrometer tumor sections were stained with either hematoxylineosin or used for immunohistochemistry (see below). The normal skin sample (Figures 1 and 3 , skin) came from the face of a 70-year-old man. The slowly growing tumor sample (Figure 3, BCC) came from the face of a 69-year-old woman. The histological analysis revealed the growth of basaloid cell solid nests at the dermoepidermal junction with diagnostic characteristics of basal cell carcinoma. The highly malignant tumor sample (Figures 1 and 3, SCC) came from the retroauricular skin of a 73-year-old man. The histological analysis showed the neoplastic growth of epithelial cells, with squamous differentiation and focal areas of acantholysis.

Tumor induction in mice. Malignant skin tumors were induced in nude (NMRI nu) mice by subcutaneous flank inoculation of $10^{6}$ PDV.C57 epidermal tumor cells. When tumors had reached an average volume of $500 \mathrm{~mm}^{3}$ (range, $400-600 \mathrm{~mm}^{3}$ ), a continuous flow pump (Alzet 2002; Alza Corporation, Palo Alto, California, USA) was surgically implanted on the flank of every mouse near the site of tumor inoculation. The pump was filled with either vehicle, $1,580 \mu \mathrm{g}$ of WIN$55,212-2$, or $1,580 \mu \mathrm{g}$ of JWH-133 in $236 \mu \mathrm{l}$ PBS supplemented with $5 \mathrm{mg} / \mathrm{ml} \mathrm{BSA}$ and operated at a flux of $0.52 \mu \mathrm{l} / \mathrm{h}$ for 11 days. Tumors were measured with an external caliper and their volume calculated as $(4 \pi / 3) \times$ $(\text { width } / 2)^{2} \times($ length $/ 2)$. Cannabinoid treatment did not affect animal weight gain (data not shown).

Viability and apoptosis in vitro. Cell viability in the cultures was determined by the 3-4,5-dimethylthiazol-2,5diphenyltetrazolium bromide thiazol blue test. Apoptosis was determined by both TUNEL staining (21) and oligonucleosomal DNA fragmentation (26) according to kit manufacturer's instructions (Boehringer Mannheim $\mathrm{GmbH}$, Mannheim, Germany).

Proliferation and apoptosis in vivo. For proliferation assays, mice received an intraperitoneal injection of BrdU $(120 \mathrm{mg} / \mathrm{kg}$ body weight) (Boehringer Mannheim $\mathrm{GmbH}) 2$ hours before tumor harvesting. Detection of BrdU-positive cells was performed using an anti-BrdU mouse $\mathrm{mAb}$ (Boehringer Mannheim $\mathrm{GmbH}$ ) as described (9). Apoptosis was determined by TUNEL 
staining (Boehringer Mannheim $\mathrm{GmbH}$ ) according to kit manufacturer's instructions (21).

Western blot analysis. Particulate cell or tissue fractions were subjected to SDS-PAGE, and proteins were transferred from the gels onto polyvinylidene fluoride membranes. To determine cannabinoid receptor expression the blots were incubated with polyclonal $\mathrm{Ab}$ 's raised in rabbits against residues 1-14 of the human/mouse $\mathrm{CB}_{1}$ receptor (1:500; kindly given by A. Howlett, North Carolina Central University, Durham, North Carolina, USA) or residues $20-33$ of the human/mouse $\mathrm{CB}_{2}$ receptor (1:2,000; Cayman Chemical, Ann Arbor, Michigan, USA) as described (21). Antigen preabsorption experiments were performed by preincubating $\left(37^{\circ} \mathrm{C}, 1\right.$ hour $1 \mu \mathrm{l}(=0.5 \mu \mathrm{g})$ of the anti- $\mathrm{CB}_{1}$ or anti$\mathrm{CB}_{2} \mathrm{Ab}$ and $100 \mu \mathrm{l} \mathrm{PBS}$ with or without $20 \mu \mathrm{l}(=20 \mu \mathrm{g})$ of the corresponding immune peptide (Cayman Chemical). Western blots were subsequently done with the aforementioned $\mathrm{Ab}$ dilutions. To determine EGF-R phosphorylation the blots were incubated with antiphosphotyrosine (1:500; 4G10 mAb; Upstate Biotechnology Inc., Lake Placid, New York, USA) or anti-keratin 5 (1:1,000; Berkeley Antibody Co., Richmond, California, USA) Ab's, the latter used as a loading control. In all cases, samples were subjected to luminography with an enhanced chemiluminescence detection kit (Amersham Life Sciences, Arlington Heights, Illinois, USA). Densitometric analysis of the blots was performed with the Molecular Analyst software package (Bio-Rad Laboratories Inc., Hercules, California, USA).

Immunohistochemistry. Tissues were fixed in $10 \%$ buffered formalin and embedded in paraffin. Sections of mouse and human skin and tumors were stained with the aforementioned $A b$ 's against $\mathrm{CB}_{1}(1: 300)$ and $\mathrm{CB}_{2}$ (1:300) receptors. Control immunostainings using the secondary $\mathrm{Ab}$ in the absence of the primary $\mathrm{Ab}$ were routinely performed. In addition, antigen preabsorption experiments were carried out with the corresponding blocking peptides as described above. Immunodetection of blood vessels in cryosections of mouse tumors was performed with an anti-CD31 Ab (1:40; PharMingen, San Diego, California, USA). Sections were incubated with a biotinylated anti-rabbit $\left(\mathrm{CB}_{1}\right.$ and $\left.\mathrm{CB}_{2}\right)$ or anti-rat $\mathrm{Ab}(\mathrm{CD} 31)$ and then with peroxidase-conjugated streptavidin (LSAB Kit Peroxidase; DAKO A/S, Glostrup, Denmark). Ab localization was determined using 3,3'-diaminobenzidine (Vector Laboratories, Burlingame, California, USA). Morphometric values were obtained by examination of six $0.11-\mathrm{mm}^{2}$ sections per tumor with the image analysis system Leica Qwin (Leica Microsystems Inc., Chantilly, Virginia, USA).

Northern blot analysis. Total RNA was extracted from the tumor samples by the acid guanidinium method (27). The VEGF probe has been described previously (7). The placental growth factor (PIGF) probe was kindly given by G. Persico (Istituto Internazionale di Genetica e Biofisica, Naples, Italy). Probes for angiopoietin 1 (Ang1) and angiopoietin 2 (Ang2) detection were kindly provided by G.D. Yancopoulos (Regeneron Pharma- ceuticals, Tarrytown, New York, USA). A 1-kb fragment of the $5^{\prime}$ region of the hEGF-R cDNA was used as probe for EGF-R detection (9). Ribosomal 7S RNA was used as a loading control. Densitometric analysis of the blots was performed with a PhosphorImager using Quantity One software (Bio-Rad Laboratories Inc.).

Statistics. Results shown represent means \pm SD. Statistical analysis was performed by ANOVA with a post hoc analysis by the Student-Neuman-Keuls test. Data in Table 1, Figure 5a, and Figure 6c were analyzed by the Mann-Whitney (Wilcoxon) $W$ test to compare medians for nonparametric data.

\section{Results}

Cannabinoid receptors are expressed in skin and skin tumors. The expression of cannabinoid receptors in epidermal cell lines, normal skin, and skin tumors of mice and humans was examined by Western blot analysis and immunohistochemistry. Western blot experiments showed that $\mathrm{CB}_{1}$ and $\mathrm{CB}_{2}$ receptors were expressed in a number of tumorigenic and nontransformed epidermal cell lines of murine and human origin (Figure 1a). In addition, the two receptors were present in normal mouse skin as well as in benign (papillomas) and malign (squamous cell carcinomas) mouse skin tumors. Likewise, $\mathrm{CB}_{1}$ and $\mathrm{CB}_{2}$ receptors were expressed in human skin, keratinocytes, and carcinomas (Figure 1a). To ascertain the specificity of the cannabinoid receptor Ab's used in the blotting experi-

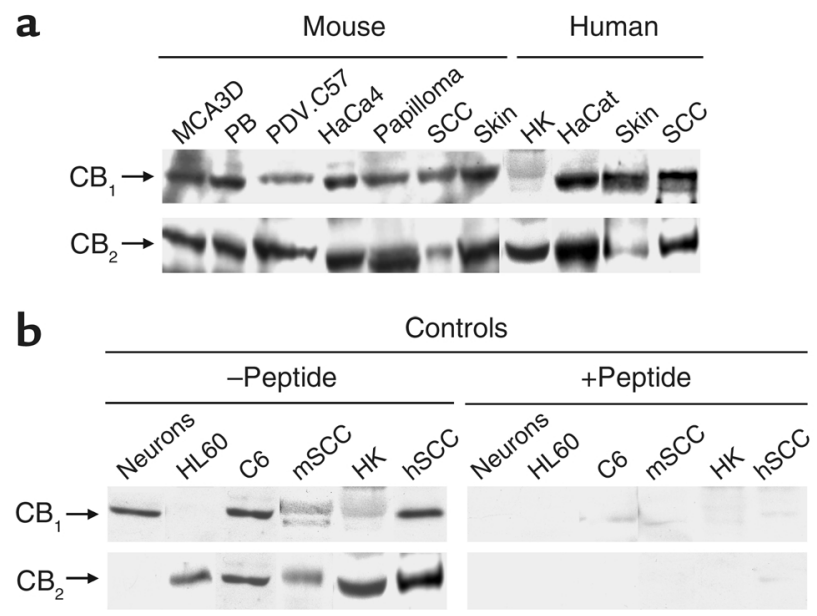

Figure 1

Western blot analysis of cannabinoid receptor expression in normal skin and skin tumors. (a) $\mathrm{CB}_{1}$ and $\mathrm{CB}_{2}$ receptor expression in murine and human epidermal cell lines, normal skin, and skin tumors. (b) Controls with the anti-CB $B_{1}$ or anti- $\mathrm{CB}_{2} \mathrm{Ab}$-blocking peptide are shown (see Methods and Results for explanation). Mouse papillomas were generated by chemical carcinogenesis (as described in ref. 9), while mouse squamous cell carcinomas (SCCs) were generated by inoculation of PDV.C57 epidermal tumor cells as described in Methods. The source of human samples is described in Methods. Images of representative samples are shown. Similar results were obtained in at least two other blots. PB, immortalized mouse nontumorigenic cell line derived from SENCAR mice papillomas; HK, human keratinocytes; $m$, mouse; h, human. 


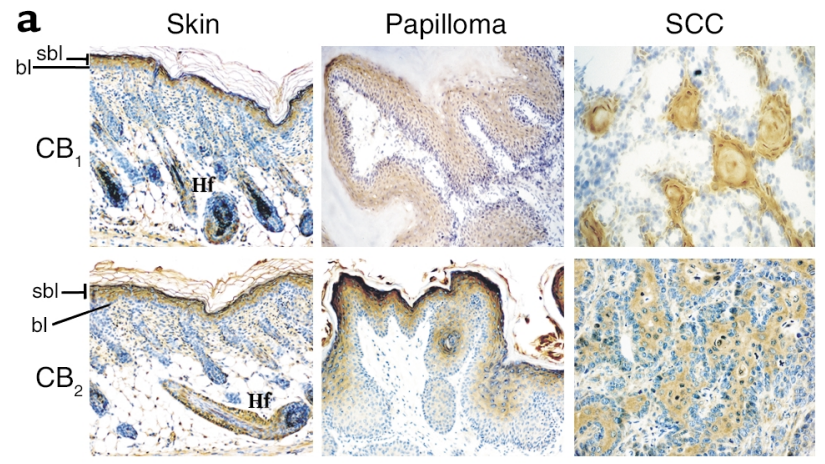

b

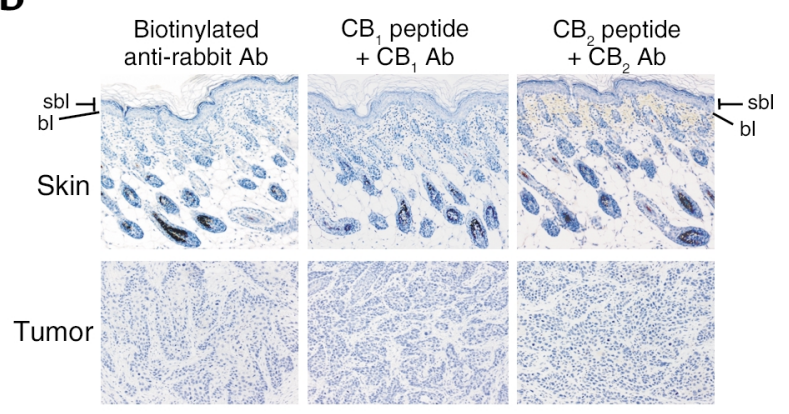

ments, antigen preabsorption experiments were carried out with the corresponding blocking peptides. As shown in Figure $1 \mathrm{~b}$, the peptides blocked anti-CB $\mathrm{CB}_{1}$ and anti- $\mathrm{CB}_{2} \mathrm{Ab}$ binding, not only in skin-derived samples but also in other cell types used as well-established controls for the presence of $\mathrm{CB}_{1}$ (rat cortical neurons) (18), $\mathrm{CB}_{2}$ (human promyelocytic HL60 cells) (19), and $\mathrm{CB}_{1}$ plus $\mathrm{CB}_{2}$ (rat $\mathrm{C} 6$ glioma cells) (21).

Immunocytochemical analyses showed that in mouse (Figure 2a) and human (Figure 3a) normal skin $\mathrm{CB}_{1}$ and $\mathrm{CB}_{2}$ receptors were mostly present in suprabasal layers of the epidermis and hair follicles. Basal staining was also observed in some sporadic regions. $\mathrm{CB}_{1}$ and $\mathrm{CB}_{2}$ receptor immunoreactivity was also evident in both papillomas and squamous cell carcinomas of mouse origin (Figure 2a), as well as in human basal cell carcinomas and squamous cell carcinomas (Figure 3a). The specificity of the immunolabeling was shown by experiments in which the primary $\mathrm{Ab}$ was omitted and-as mentioned above for Western blots-by antigen preabsorption experiments carried out with the corresponding blocking peptides (Figures $2 \mathrm{~b}$ and $3 \mathrm{~b}$ ).

Cannabinoid receptor activation induces skin tumor cell apoptosis. We tested the functionality of cannabinoid

\section{Figure 3}

Immunohistochemical analysis of cannabinoid receptor expression in human normal skin and skin tumors. (a) Immunolocalization of $C_{1}$ and $C_{2}$ receptors. (b) Controls without primary $A b$ (only with secondary biotinylated anti-rabbit $A b$ ), as well as controls with the Ab-blocking peptides, are shown (see Methods and Results for explanation). The source of samples is described in Methods. BCC, basal cell carcinoma; SCC, squamous cell carcinoma; Hf, hair follicle.

\section{Figure 2}

Immunohistochemical analysis of cannabinoid receptor expression in mouse normal skin and skin tumors. (a) Immunolocalization of $C_{1} B_{1}$ and $C_{2}$ receptors. (b) Controls without primary $A b$ (only with secondary biotinylated anti-rabbit $A b$ ), as well as controls with the Ab-blocking peptides, are shown (see Methods and Results for explanation). Normal skin came from a 5-day-old mouse. Papillomas were generated by chemical carcinogenesis (as described in ref. 9), while SCCs were generated by inoculation of PDV.C57 epidermal tumor cells as described in Methods. Images of representative samples are shown. Similar results were obtained in at least two other samples. $\mathrm{Hf}$, hair follicle; bl, basal layer; sbl, suprabasal layers.

receptors in the induction of apoptosis in skin tumor cells. The mixed $\mathrm{CB}_{1} / \mathrm{CB}_{2}$ agonist WIN-55,212-2 decreased the viability of the tumorigenic epidermal cell lines PDV.C57 and HaCa4 (Figure 4a). Values of cell viability after WIN-55,212-2 treatment (as percentage of the corresponding incubations with no additions) were $74 \pm 10$ (day 3 ) and $62 \pm 7$ (day 4) for PDV.C57 cells, and $75 \pm 7$ (day 3 ) and $71 \pm 4$ (day 4) for $\mathrm{HaCa} 4$ cells. Of interest, the cannabinoid was unable to induce any statistically significant change in the viability of MCA3D and HaCat cells, two nontransformed epidermal cell lines, and of primary human keratinocytes (Figure $4 \mathrm{~b}$ ). The mixed $\mathrm{CB}_{1} / \mathrm{CB}_{2}$ agonist HU-210 and the selective $\mathrm{CB}_{2}$ agonist JWH-133 $\left(K_{\mathrm{i}}=677 \mathrm{nM}\right.$ for $\mathrm{CB}_{1}$ and $3.4 \mathrm{nM}$ for $\mathrm{CB}_{2}$; ref, 25) (both at $25 \mathrm{nM}$ ) also induced PDV.C57 death to an extent similar to that of WIN-55,212-2 (data not shown). WIN-55,212-2-induced death of PDV.C57 cells occurred by a process of apoptosis, as determined by oligonucleosomal DNA fragmentation (Figure 4c) and TUNEL staining (Figure 4d).

To evaluate the possible implication of the $\mathrm{CB}_{1}$ and $\mathrm{CB}_{2}$ receptors in cannabinoid-induced apoptosis, the

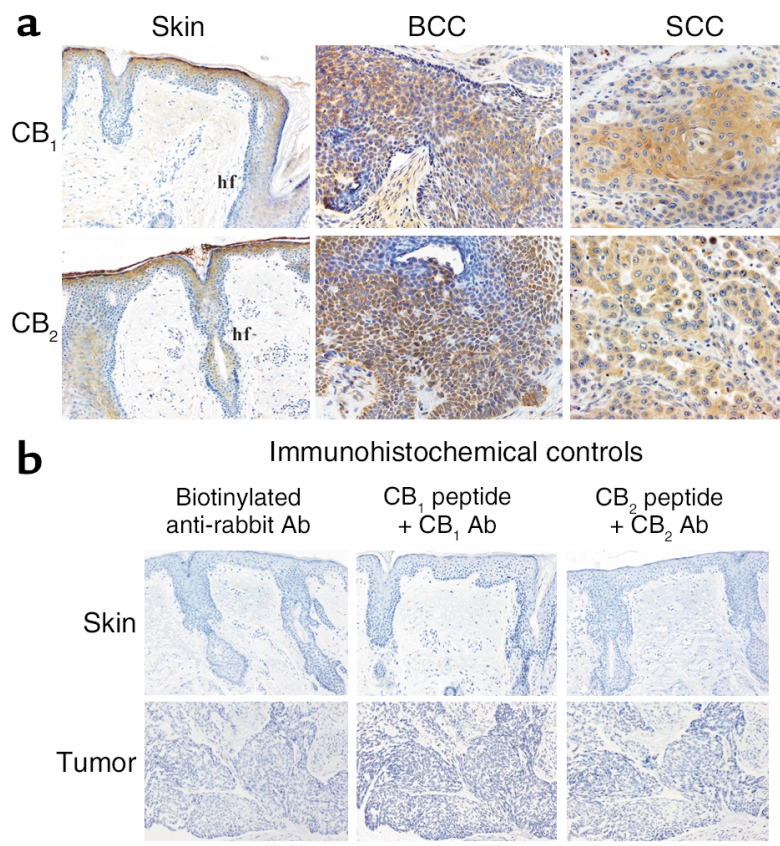



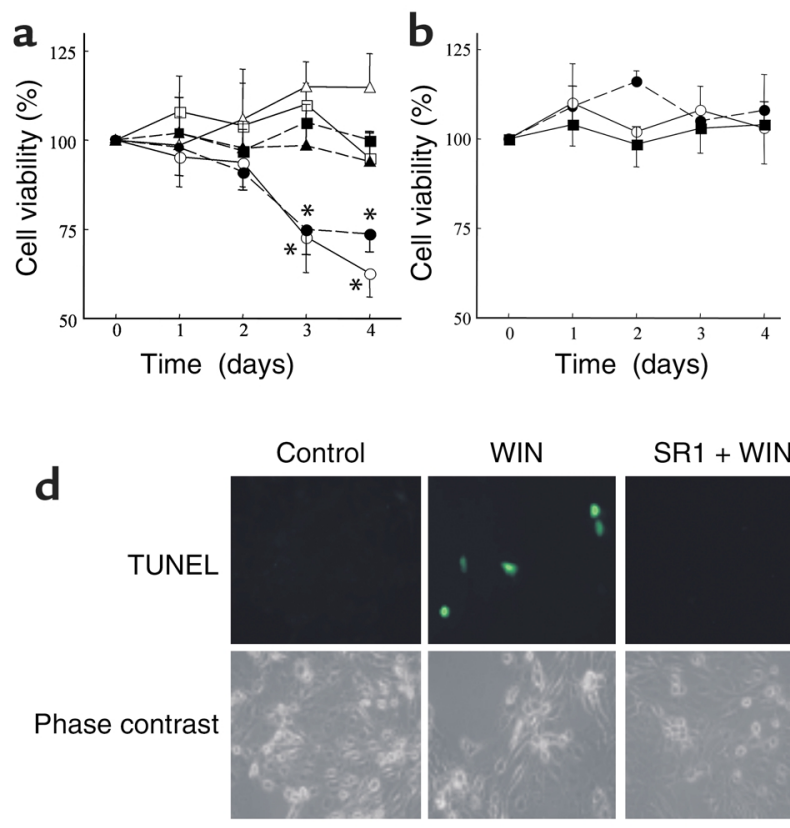

WIN
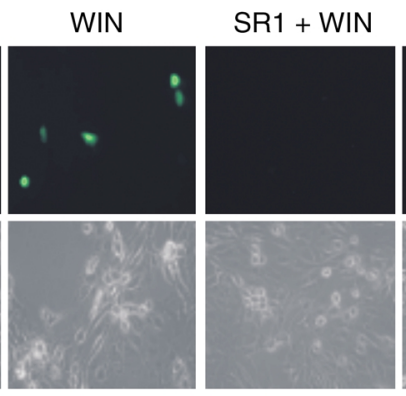

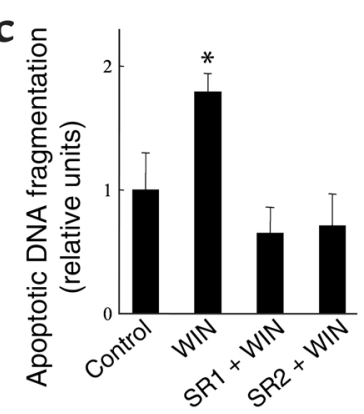

SR2 + WIN
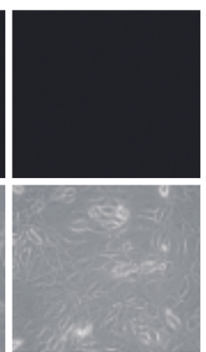

\section{Figure 4}

Cannabinoid receptor activation induces skin tumor cell apoptosis. (a) The tumorigenic epidermal cell lines PDV.C57 (open symbols) and $\mathrm{HaCa} 4$ (closed symbols) were cultured with $25 \mathrm{nM}$ WIN-55,212-2 alone (circles), or in combination with 0.2 $\mu \mathrm{M}$ SR141716 (SR1) (squares), or $0.2 \mu \mathrm{M}$ SR144528 (SR2) (triangles), and cell viability was determined $(n=5)$. (b) The nontransformed epidermal cell lines MCA3D (open circles) and HaCat (closed circles), as well as primary human keratinocytes (closed squares), were cultured with $25 \mathrm{nM}$ WIN-55,212-2, and cell viability was determined $(n=4)$. (c and d) PDV.C57 cells were cultured as described before, and oligonucleosomal DNA fragmentation (c) $(n=6)$, and TUNEL staining (d) (one representative experiment of four) were assessed. ${ }^{*}$ Significantly different $(P<0.01)$ from control incubations. effect of selective receptor antagonists was studied. Thus, the $\mathrm{CB}_{1}$ antagonist SR141716 and the $\mathrm{CB}_{2}$ antagonist SR144528 prevented WIN-55,212-2induced apoptosis of PDV.C57 cells (Figures 4, a, c, and d), pointing to the involvement of both receptors in the apoptotic action of cannabinoids.

Cannabinoids inhibit skin tumorgrowth in vivo. Given the inhibition of tumorigenic epidermal cell survival in culture by cannabinoids, we evaluated the effect of cannabinoid treatment on skin tumor growth in vivo. Tumors generated by inoculation of the highly malignant PDV.C57 cell line were treated with vehicle or WIN-55,212-2. As shown in Figure 5, a-c, cannabinoid administration blocked the growth of tumor cells in vivo (in $\approx 75 \%$ of the mice treated).

Because cannabinoid-based therapeutic strategies should be as devoid as possible of psychotropic side effects and PDV.C57 express functional $\mathrm{CB}_{2}$ receptors, we administered to mice the selective $\mathrm{CB}_{2}$ agonist JWH133. Previously, we have provided pharmacological, biochemical, and behavioral evidence that JWH-133 activates selectively the $\mathrm{CB}_{2}$ receptor and does not elicit psychotropic effects in mice (22). As shown in Figure 5 , a-c, tumors from JWH-133-treated animals were significantly smaller than those from vehicle-treated controls (in $\approx 70 \%$ of the mice treated).

We next examined whether, as occurs in cultured skin tumor cell lines, cannabinoids induce apoptosis of malignant cells in vivo. As shown in Table 1, quantification of apoptotic cells in tumor sections revealed that treatment with WIN-55,212-2 or JWH-133 increased the number of apoptotic cells. In contrast, the proliferation index did not significantly differ between control and cannabinoid-treated carcinomas.

Cannabinoids inbibit skin tumor angiogenesis in vivo. Tumors require an adequate supply of oxygen and nutrients to grow more than a few millimeters. For that purpose they produce proangiogenic factors that promote the formation of new blood vessels $(28,29)$. We therefore analyzed whether the vascularization of growth-arrested cannabinoid-treated tumors was affected. Northern blot analyses showed that the

\section{Figure 5}

Cannabinoids inhibit skin tumor growth in vivo. PDV.C57 cells were injected subcutaneously in mice. When tumors had reached the desired size, animals were treated with either vehicle (Co), WIN-55,212-2 (WIN), or JWH-133 (JWH), for 11 days. (a) Tumor size ( $n=8$ for each experimental group). *Significantly different $(P<0.01)$ from control mice. (b) Examples of subcutaneous tumors in the flank of mice after the indicated treatments. (c) Appearance of tumors dissected from mice after the indicated treatments.

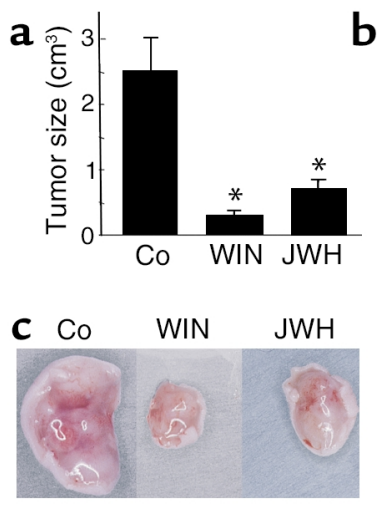

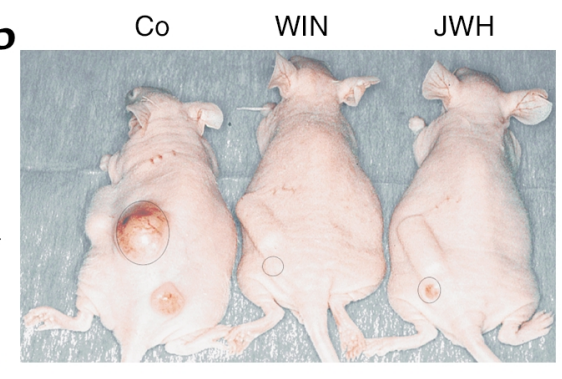



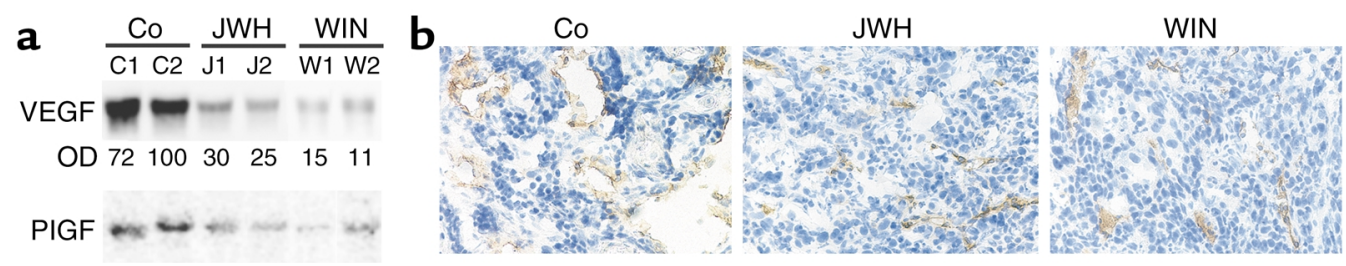

OD $91 \quad 100 \quad 62 \quad 33 \quad 14 \quad 41$
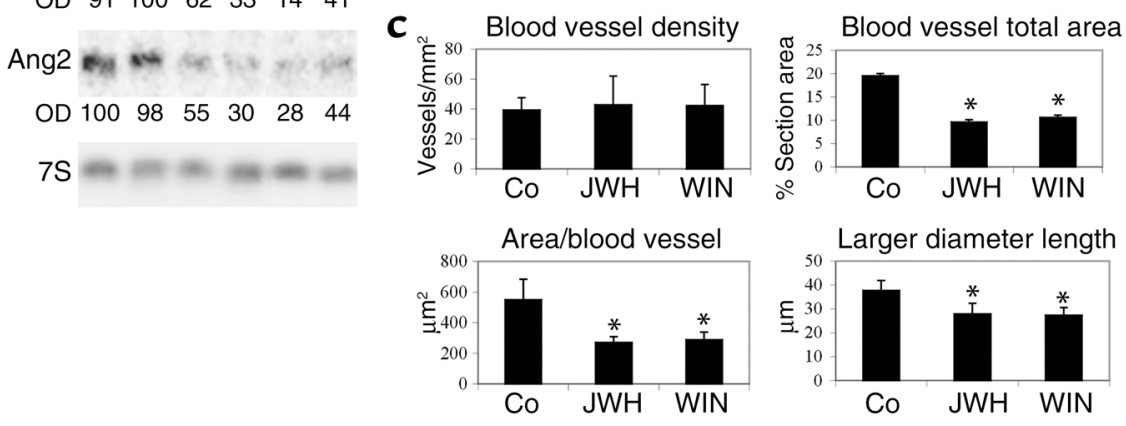

\section{Figure 6}

Cannabinoids inhibit angiogenesis in skin tumors in vivo. PDV.C57 cells were injected subcutaneously in mice. When tumors had reached the desired size, animals were treated with either vehicle (Co), WIN-55,212-2 (WIN,) or JWH-133 (JWH) for 11 days. (a) Northern blot of the proangiogenic factors VEGF, PIGF, and Ang2. C1, C2; J1, J2; W1, W2 designate tumors from two different animals of each experimental group, that is, treated with vehicle (Co), JWH-133, or WIN-55,212-2, respectively. OD values relative to those of loading controls are given in arbitrary units. 7S, Ribosomal 7S RNA. (b) CD31 immunostaining. Note that control carcinomas show dilated blood vessels, while vessels of cannabinoid-treated tumors are narrow. (c) Morphometric analysis of tumor vasculature $(n=4-6$ for each experimental group). *Significantly different $(P<0.05)$ from control mice.

expression of major proangiogenic factors, namely VEGF, PIGF, and Ang2, was strongly depressed by treatment with WIN-55,212-2 or JWH-133 (Figure 6a). The mRNA expression of Ang1 and the two antiangiogenic factors, thrombospondin 1 and thrombospondin 2 , was not significantly affected by cannabinoid administration (data not shown). Furthermore, although immunostaining of CD31, a marker of endothelial cells, revealed no significant differences in vascular density (number of blood vessels per unit area) between control and WIN-55,212-2- or JWH-133-treated tumors (Figures 6, b and c), important differences were observed when vessel morphology was examined: while control carcinomas showed a network of dilated vessels, cannabinoid-treated tumors displayed a pattern of blood vessels characterized predominantly by narrow capillaries (Figure 6b). Morphometric analyses con- firmed that cannabinoid treatment induced a statistically significant decrease in blood vessel size, as determined by the total area occupied by vessels, the area per vessel, and the vessel larger diameter length (Figure 6c).

Cannabinoids decrease EGF-R activation in skin tumors in vivo. We have recently found that in skin carcinomas EGF-R plays an important role in triggering the angiogenic switch necessary for skin tumor growth (9). Thus, we measured the expression levels and activation state of EGF-R in control and cannabinoid-treated skin tumors. While EGF-R mRNA was highly expressed in vehicletreated tumors, in line with its known overexpression in skin carcinomas $(30,31)$, the levels of EGF-R mRNA in cannabinoid-treated tumors were very low (Figure 7a). In addition, the degree of EGF-R activation (autophosphorylation) was markedly reduced in cannabinoidtreated tumors (Figure 7b). Moreover, exposure of cul-

\section{Figure 7}

Cannabinoids inhibit EGF-R activation in skin tumors in vivo. (a and $\mathbf{b}$ ) PDV.C57 cells were injected subcutaneously in mice. When tumors had reached the desired size, animals were treated with either vehicle (Co), WIN55,212-2, or JWH-133 for 11 days. Northern (a) and Western blot (b) analyses show that EGF-R mRNA expression and EGF-R activation (autophosphorylation), respectively, are severely diminished in cannabinoid-treated tumors. One representative experiment of three is shown in each panel. (c) PDV.C57 cells were cultured for 24 hours with either vehicle, $25 \mathrm{nM}$ WIN$55,212-2$, or $25 \mathrm{nMJWH}-133$, and EGF-R phosphorylation was determined by Western blot analysis. One representative experiment of three is shown. $O D$ values relative to those of loading controls are given in arbitrary units. Ribosomal 7S RNA (7S) and keratin 5 (K5) were used as loading controls in Northern and Western blots, respectively. PY, phosphotyrosine.
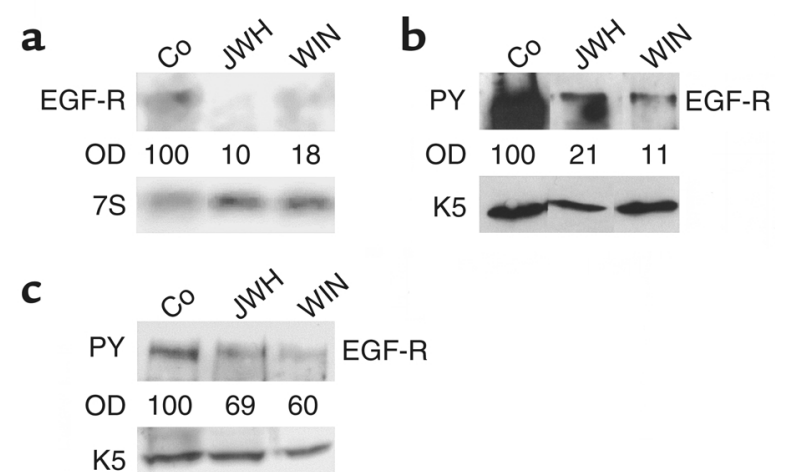
tured PDV.C57 cells to WIN-55,212-2 or JWH-133 blunted EGF-R phosphorylation (Figure 7c), supporting the direct impact of cannabinoids on skin tumor cells.

\section{Discussion}

Here we report that $\mathrm{CB}_{1}$ and $\mathrm{CB}_{2}$ cannabinoid receptors are expressed in normal epidermis and in skin tumors and that both receptors are functional in the induction of apoptosis of skin tumor cells and the regression of skin carcinomas. It is therefore plausible that apoptosis of tumor cells and tumor regression are two causally related events. Nonetheless, our data indicate that cannabinoid antitumoral action may also rely on the inhibition of tumor angiogenesis. It has been shown that mouse skin tumor growth and progression depends on critical events leading to epithelial and stromal changes, including the establishment of an active angiogenesis (4). Here, we report that blood vessels developed by cannabinoid-treated carcinomas are small, in line with the finding that blood vessel enlargement constitutes a prominent feature of skin tumor progression $(4,32)$. Moreover, we show that in cannabinoid-treated carcinomas the expression of proangiogenic factors is depressed and that of antiangiogenic factors is unchanged, which fits well with the observations that link skin carcinoma development with a clear imbalance toward positive angiogenic-factor action $(6,7,9)$. Ha-ras activation seems to be a critical event in mouse skin tumor initiation as well as a major component of the angiogenic response (6) in which VEGF plays a pivotal role $(5,9)$. Ha-ras activation induces VEGF expression in mouse keratinocytes (6), as well as in other cell types $(33,34)$. Our data also show that cannabinoid treatment decreases the expression of PIGF (another VEGF family member) and Ang2, and these two proangiogenic factors may act in concert with VEGF because their expression is highly increased since the early stages of tumor development $(9,28,29)$. EGF-R participates in the regulation of key epidermal functions (35-38). Moreover, we have shown that in mouse skin carcinomas EGF-R-dependent Ha-ras activation plays a pivotal role in VEGF expression and tumor angiogenesis and growth (9). Carcinoma growth arising from subcutaneous injection of tumor epidermal cells is a biphasic process. The first phase of slow growth occurs independently of EGF-R function. Later, an angiogenic switch response mediated by the EGF-R seems to be an essential requirement for complete tumor growth, involving high VEGF levels. Other members of the EGF-R family such as HER2 may also exert their relevant anticarcinogenic role via modulation of angiogenesis (39). Here we show that cannabinoid treatment impairs EGF-R function, VEGF expression, and angiogenesis in skin tumors. It is of interest that inhibition of EGF-R function also occurred upon exposure of cultured skin tumor cells to cannabinoids, indicating that the changes observed in EGF-R activity in vivo reflect a direct impact of cannabinoids on tumor cells and are not a mere consequence of decreased tumor size. Although at present we cannot establish the mechanism for the decrease of EGF-R phosphorylation in cannabinoid-treated tumors, it is tempting to speculate that cannabinoid treatment interferes with the tumor angiogenic switch and that this, together with the direct induction of apoptosis on tumor cells, is a reason for the inhibition of tumor growth in our system.

Nonmelanoma skin cancer is one of the most common malignancies in humans. Different types of strategies are currently being investigated as therapies for the treatment of these tumors, including cryotherapy, topical chemotherapeutic agents such as 5-fluorouracil, and photodynamics, the success of which is hampered by limitations such as the poor penetration of molecules into the skin and the difficulty of gaining access to the whole tumor (10-12). The present data indicate that local cannabinoid administration may constitute an alternative therapeutic approach for the treatment of nonmelanoma skin cancer. Of further therapeutic interest, we show that skin cells express functional $\mathrm{CB}_{2}$ receptors. The synergy between $\mathrm{CB}_{1}$ and $\mathrm{CB}_{2}$ receptors in eliciting skin tumor cell apoptosis reported here is nonetheless intriguing because it is not observed in the case of cannabinoid-induced glioma cell apoptosis (21, 22). In any event, the present report, together with the implication of $\mathrm{CB}_{2}$ - or $\mathrm{CB}_{2}$-like receptors in the control of peripheral pain (40-42) and inflammation (41), opens the attractive possibility of finding cannabinoidbased therapeutic strategies for diseases of the skin and other tissues devoid of nondesired $\mathrm{CB}_{1}$-mediated psychotropic side effects.

\section{Acknowledgments}

We are indebted to M.I. de los Santos for expert technical assistance and to F. Larcher for discussion and advice. This work was supported by grants from the Ministerio de Ciencia y Tecnología (PM 98-0079 to M. Guzmán, SAF 98-0047 to J.L. Jorcano, and BMC 20011018 to J.L. Jorcano); the Comunidad Autónoma de Madrid (08.1/0079/2000 to M. Guzmán); the Fundación Ramón Areces (to M. Guzmán); and the National Institute on Drug Abuse (DA03590 to J.W. Huffman).

1. Fuchs, E. 1990. Epidermal differentiation: the bare essential. J. Cell Biol. 11:2807-2814.

2. Limmer, B.L. 2001. Nonmelanoma skin cancer: today's epidemic. Tex. Med. 97:56-58.

3. Morris, R.J., Tryson, K.A., and Wu, K.Q. 2000. Evidence that the epidermal targets of carcinogen action are found in the interfollicular epidermis of infundibulum as well as in the hair follicles. Cancer Res. 60:226-229.

4. Bolontrade, M.F., et al. 1998. Angiogenesis is an early event in the development of chemically induced skin tumors. Carcinogenesis. 19:2107-2113.

5.Zhang, H.T., et al. 1995. Enhancement of tumor growth and vascular density by transfection of vascular endothelial cell growth factor into MCF-7 human breast carcinoma cells. J. Natl. Cancer Inst. 87:213-219.

6. Larcher, F., et al. 1996. Up-regulation of vascular endothelial growth factor/vascular permeability factor in mouse skin carcinogenesis correlates with malignant progression state and activated $\mathrm{H}$-ras expression levels. Cancer Res. 56:5391-5396.

7. Larcher, F., Murillas, R., Bolontrade, M., Conti, C.J., and Jorcano, J.L. 1998. VEGF/VPF overexpression in skin of transgenic mice induces angiogenesis, vascular hyperpermeability and accelerated tumor development. Oncogene. 17:303-311. 
8. Sibilia, M., et al. 2000. The EGF receptor provides an essential survival signal for SOS-dependent skin tumor development. Cell. 102:211-220.

9. Casanova, M.L., et al. 2002. A critical role for ras-mediated, EGFRdependent angiogenesis in mouse skin carcinogenesis. Cancer Res. 62:3402-3407.

10. Skidmore, R.A., and Flowers, F.P. 1998. Nonmelanoma skin cancer. Med. Clin. North Am. 82:1309-1323.

11. Leber, K., Perron, V.D., and Sinni-McKeehen, B. 1999. Common skin cancers in the United States: a practical guide for diagnosis and treatment. Nurse Pract. Forum. 10:106-112.

12. De Rosa, F.S., and Bentley, M.V. 2000. Photodynamic therapy of skin cancers: sensitizers, clinical studies and future directives. Pharm. Res. 17:1447-1455.

13. Pertwee, R.G. 2000. Cannabinoid receptor ligands: clinical and neuropharmacological considerations, relevant to future drug discovery and development. Exp. Opin. Investig. Drugs. 9:1-19.

14. Piomelli, D., Giuffrida, A., Calignano, A., and Rodríguez de Fonseca, F. 2000. The endocannabinoid system as a target for therapeutic drugs. Trends Pharmacol. Sci. 21:218-224.

15. Porter, A.C., and Felder, C.C. 2001. The endocannabinoid nervous system: unique opportunities for therapeutic intervention. Pharmacol. Ther 90:45-60.

16. Devane, W.A., et al. 1992. Isolation and structure of a brain constituent that binds to the cannabinoid receptor. Science. 258:1946-1949.

17. Mechoulam, R., et al. 1995. Identification of an endogenous 2-monoglyceride, present in canine gut, that binds to cannabinoid receptors. Biochem. Pharmacol. 50:83-90.

18. Matsuda, L.A., Lolait, S.J., Brownstein, M., Young, A., and Bonner, T.I. 1990. Structure of a cannabinoid receptor and functional expression of the cloned cDNA. Nature. 346:561-564.

19. Munro, S., Thomas, K.L., and Abu-Shaar, M. 1993. Molecular characterization of a peripheral receptor for cannabinoids. Nature. 365:61-65

20. Munson, A.E., Harris, L.S., Friedman, M.A., Dewey, W.L., and Carchman, R.A. 1975. Antineoplastic activity of cannabinoids. J. Natl. Cancer Inst. 55:597-602.

21. Galve-Roperh, I., et al. 2000. Anti-tumoral action of cannabinoids: Involvement of sustained ceramide accumulation and extracellular signal-regulated kinase activation. Nat. Med. 6:313-319.

22. Sánchez, C., et al. 2001. Inhibition of glioma growth in vivo by selective activation of the $\mathrm{CB}_{2}$ cannabinoid receptor. Cancer Res. 61:5784-5789.

23. Bifulco, M., et al. 2001. Control by the endogenous cannabinoid system of ras oncogene-dependent tumor growth. FASEB J. 15:2745-2747.

24. Guzmán, M., Sánchez, C., and Galve-Roperh, I. 2001. Control of the cell survival/death decision by cannabinoids. J. Mol. Med. 78:613-625.

25. Huffman, J.W., et al. 1999. 3-(1',1'-Dimethylbutyl)-1-deoxy-delta-8THC and related compounds: synthesis of selective ligands for the
$\mathrm{CB}_{2}$ receptor. Bioorg. Med. Chem. Lett. 7:2905-2914.

26. Blázquez, C., Galve-Roperh, I., and Guzmán, M. 2000. De novo-synthesized ceramide signals apoptosis in astrocytes via extracellular signalregulated kinase. FASEB J. 14:2315-2322.

27. Chomczynski, P., and Sacchi, N. 1987. Single-step method of RNA isolation by acid guanidinium thiocyanate-phenol-chloroform extraction. Anal. Biochem. 162:156-159.

28. Carmeliet, P., and Jain, R.K. 2000. Angiogenesis in cancer and other diseases. Nature. 407:249-257.

29. Yancopoulos, G.D., et al. 2000. Vascular-specific growth factors and blood vessel formation. Nature. 407:242-248.

30. Hunts, J., et al. 1985. Hyperproduction and gene amplifications of the EGFR in squamous cell carcinomas. Jpn. J. Cancer Res. 76:633-666.

31. Rho, O., Beltran, L.M., Conti-Giménez, I.B., and DiGiovanni, J. 1994 Altered expression of the epidermal growth factor receptor and transforming growth factor-alpha during multistage skin carcinogenesis in SENCAR mice. Mol. Carcinog. 11:19-28.

32. Hawighorst, T., et al. 2001. Thrombospondin-2 plays a protective role in multistep carcinogenesis: a novel host anti-tumor defense mechanism. EMBO J. 20:2631-2640.

33. Grugel, S., Finkenzeller, G., Weindel, K., Barleon, B., and Marme, D. 1995. Both v-Ha-Ras and v-Raf stimulate expression of the vascular endothelial growth factor in NIH 3T3 cells. J. Biol. Chem. 270:25915-25919.

34. Rak, J., et al. 1995. Mutant ras oncogenes upregulate VEGF/VPF expression: implications for induction and inhibition of tumor angiogenesis. Cancer Res. 55:4575-4580.

35. Luetteke, N.C., et al. 1993. TGF alpha deficiency results in hair follicle and eye abnormalities in targeted and waved-1 mice. Cell. 73:263-278.

36. Luetteke, N.C., et al. 1994. The mouse waved-2 phenotype results from a point mutation in the EGF receptor tyrosine kinase. Genes Dev. 8:399-413.

37. Murillas, R., Larcher, F., Conti, C.J., Santos, M., and Jorcano, J.L. 1995. Expression of a dominant negative mutant of epidermal growth factor receptor in the epidermis of transgenic mice elicits striking alterations in hair follicle development and skin structure. EMBO J. 14:5216-5223.

38. Jost, M., Kari, C., and Rodeck, U. 2000. The EGF receptor-an essential regulator of multiple epidermal functions. Eur. J. Dermatol. 10:505-510.

39. Izumi, Y., Xu, L., Di Tomaso, E., Fukumura, D., and Jain, R.K. 2002. Herceptin acts as an anti-angiogenic cocktail. Nature. 416:279-280.

40. Calignano, A., La Rana, G., Giuffrida, A., and Piomelli, D. 1998. Control of pain initiation by endogenous cannabinoids. Nature. 394:277-281

41. Hanus, L., et al. 1999. HU-308: a specific agonist for $\mathrm{CB}_{2}$, a peripheral cannabinoid receptor. Proc. Natl. Acad. Sci. USA. 96:14228-14233.

42. Malan, T.P., Jr., et al. 2001. $\mathrm{CB}_{2}$ cannabinoid receptor-mediated peripheral antinociception. Pain. 93:239-245. 